\title{
STUDY ON HOW THE TELEMEDICINE HAS MADE IT EFFECTIVE DURING THIS PANDEMIC, COVID -19
}

\author{
Krushna.v \\ Master of Hospital Administration $2^{\text {nd }}$ year \\ Department of Hospital Management Studies \& \\ Staff Training \& Development \& Christian Medical College, Vellore
}

\begin{abstract}
The covid19 pandemic has made the conditions around the world pathetic, It has made the lives of the people around the world a serious question mark, the disease on the one hand and ultimately making the daily lives of the people miserable. It has given a serious blow to all the industries and job around the world. Due to the pandemic condition and it's viral spread even the normal people are hesitant to visit the hospitals around them, Therefore this study discusses about the telemedicine and its effectiveness during this pandemic situation making people aware and making hospitals all over to use this telemedicine in treating the patients, how patients can get their clinicians support without visiting the hospitals by using this telemedicine, Finally the study was performed by collecting and analysing relevant secondary data. It has also given suggestions that it is essential to make the people aware about the telemedicine just like making the clinicians aware about the working of the telemedicine.
\end{abstract}

Key Words: telemedicine, social distancing, quarantine, cost effective , E-prescription, Covid-19

\section{INTRODUCTION}

The covid19, It was previously called as the 2019 novel corona virus, since it's a new strain that has not been previously identified in humans though it has been said that it is genetically related to the earlier pandemic SARS. The virus that is causing this disease is the severe acute respiratory syndrome corona virus 2 (SARS-CoV-2) . The disease has been renamed as corona virus disease-2019. The prevalent condition everywhere right now is the lockdown as it is said that only by preventive measures this disease could be controlled as there is no evident therapies or vaccination found so far. The preventive measures followed so far are the social distancing ( $1 \mathrm{~m}$ distance), quarantining and hygienic measures. It is also said that the disease could be controlled by immunizing an Individual or by having a good immune system, making themselves resistant towards the disease. Since it's a communicable disease and the spread is fast, people have locked themselves down, staying wherever they are, which is a good thing but how long can this situation continue no one knows exactly about the destination ahead. Due to people staying at home and the fear towards the disease has made them stay away from various basic day today activities, which is an Inter dependant chain as most of the industries, groceries and other day-to-day activities runs on the basis of people's movement. This is not only the case even the patients who are supposed to take regular checkups have refrained themselves from visiting the hospitals, That makes it even worse, Medical organizations around the world had to address this kind of situation in which one such alternatives found to be was this telemedicine, still many hospitals, clinicians have not followed this method so far and the patients are also not aware too. So this paper discusses about the telemedicine and the various parameters linked with it.

\section{SUBSECTION}

\section{A.telemedicine}

B.Effective services provided by the telemedicine as outpatients services remain closed

C. Various medical Organizations on telemedicine

\section{D.Actual Diagnosis and telemedicine}

E. confidentality of patients and telemedicine

F. People's view on the telemedicine

G.Tele medicine Vs Make shift field hospital

\section{A.telemedicine}

It is a modem technology that has made clinicians to interact with their patients virtually using video conferencing tools. It was found to be used initially because of its cost-effective purpose. The access to healthcare facility has been made easier in the remote or the rural areas, where the patients need not travel so far for checkups. Telemedicine is not only two way interaction but it has also made the clinicians to provide e-prescriptions for the patients

B.Effective services provided by the telemedicine as outpatients services remain closed

As social distancing and quarantining are the only ways to control the disease so far, tele medicine has made it possible. In recent studies it has been mentioned that telemedicine is not only for the general patients but also for the covid patients who can access this technology by taking care of themselves and others even when quarantined. The telemedicine has largely helped the pregnant women with hyperglycemia or hypertension by enabling them to access the system by staying wherever they are. It has been reported that a few suspected covid cases have been detected through the telephonic 
counseling mode and were asked to visit the nearest government general hospital for screening .

\section{Various medical Organizations on telemedicine}

The consultation through telemedicine in India is by a registered Medical Practitioner under the Indian Medical Council Act, 1956.in accordance with the guidelines specified. The telemedicine is not applicable to the use of digital technology in performing surgical or invasive procedure remotely. The registered medical practitioners are supposed to follow the same professional and ethical norms and standards as done in the traditional in person care. It has been reported that through telemedicine patients records can be maintained and the connection between the doctors and the patients could be maintained . The medical organizations are also working on how to improve the standards, quality and their guidelines from time to time, finally the telemedicine can take over the primary hospitals and lessen the burden for the secondary hospitals.

\section{D.Actual Diagnosis and telemedicine}

Actual Diagnosis of a person is not possible by telemedine though clinicians could predict the diseases and their symptoms through telecounselling mode, actual diagnosis is not possible. Since actual diagnosis is not possible through telemedicine the patient can only be a regular patient to the clinician having a medical record so that at least regular checkups can be done.

\section{E. confidentality of patients and telemedicine}

As telemedicine will be practiced, it will bring with it various medicolegal problems like any other medical field. Problems of confidentiality of the patients, standards of treatment, credentialing of doctors, and consent from the patients, misconduct, licensure, reimbursement, professional negligence, duties, penalties and legal liability will have to be solved. Due to absence of any statutes or laws, especially relating to the issues this problem will be further complicated. Every individual has a right to privacy and this includes rights in relation to telemedicine. There are more chances that there can be leakage of privacy of patients as all records are computer based. Searches are very convenient and any person can have data access of any patient if there is carelessness in file addition and deletion security.

\section{F.Tele medicine Vs Make shift field hospital}

Make shift field hospitals are considered to be a temporary hospital or a mobile medical unit, In conditions like this pandemic a Make shift hospital could be effective as it's purpose is made only to address a particular situation here it's the covid disease because of which segregation of covid treating doctors and the regular medical practitioner could be done easily, even the regular patients won't be hesitant to visit the mother hospitals. Therefore Make shift field Hospital is a very good measure in this kind of pandemic, also protecting the Mother hospitals by protecting it's normal patients and from revenue loses. Though treatment cure and diagnosis and other parameters are better with the make shift field hospitals it can also be costly and making of one proper make shift field hospital can be a tedious process as it involves parameters like site selection, layout planning etc. As it is mentioned that quarantining and Social distancing are the only preventive measures so far, it could be difficult to practice social distancing with a make shift hospital. Therefore in this kind of pandemic tele medicine stands one step higher than the make shift hospitals

\section{G. People's view on the telemedicine}

From recent records it has been found that $66 \%$ of the people are willing to use the telemedicine, the usage and preference towards telemedicine varies greatly by the ages of the people. Due to lockdowns and Quarantining the millennial are found to be more stressed than before, they prefer tele-counselling to address their growing mental healthcare issues, the older population on the other hand prefer tele medicine for renewing their prescriptions and chronic care management, the middle aged people are preferring tele medicine for urgent care. Therefore it has been recorded that the younger population are more willing to adopt towards the telemedicine. The gap between the willingness and usage

From recent records it has been found that only $20 \%$ of people have heard of the term telemedicine and have used them before. After explaining about the usage of telemedicine more people have been willing to use them.

\section{DISCUSSIONS}

This study has highlighted on how covid pandemic has made telemedicine an effective thing, pandemics like these pose challenges and makes the healthcare sector to be seen in a different perspective. Tele medicine has its own flows and flaws like all the technologies have, It is not only cost effective but in this type of pandemic where social distancing and quarantining are the only preventive measures followed to control the disease, telemedicine has made it possible to some extent and we can expect in the near future that the medical organizations in and around India would keep making changes in the telemedicine until the flaws of this system is minimized to a greater extent. It also essential to make the people more aware about the technology available and explain them about the usage just like making the clinicians aware and more professional towards the using of telemedicine. Online surveys could be conducted in making the people aware about the telemedicine, Ads and social media posts on various platforms can be other days, Ads can be made in such a way that it only talks on the working of telemedicine during this pandemic and not any other kind of promotional Ads for a specific organization or a clinician.

\section{REFERENCES}

[1] Christopher; Parker, Helen; Singh, D; Wade, Elizabeth,(2010),Making the shift from hospital to the community: lessons from an evaluation of a pilot programme Ham,Primary Health Care Research \& Development,299-309.

[2]Devin M. Mann, MD, MS1,2; Ji Chen, MS1,2; Rumi Chunara, PhD3,4; Paul A. Testa, MD2; Oded Nov, PhD, MS5,(2020),COVID-19 transforms health care through 
telemedicine: evidence from the field,Journal of the American Medical Informatics Association,1132-1135.

[3]Mehrotra A, Ray K, Brockmeyer DM, Barnett ML, Bender JA, (2020), Rapidly Converting to "Virtual Practices": Outpatient Care in the Era of Covid-19. Catalyst non-issue content,NEJM Catalyst,104-106

[4] R Ohannessian,TA Duong,(2020),Global telemedicine implementation and integration within health systems to fight the Covid19 pandemic: a call to action,JMIR public health,233-236

[5] Judd E Hollander, Brendan G Carr , (2020), Virtually perfect? Telemedicine for covid19, new england journal of medicine,112-115.

[6] Bobak Moazzami, Niloofar Razavi-Khorasani,Arash Dooghaie Moghadam, Ermia Farokhi,(2020),Covid19 and telemedicine Immediate action required for maintaining healthcare providers well-being,Journal of Clinical Virology100-105.

[7] Rashid Bashshur, Charles R Doarn, Julio M Frenk, Joseph C Kvedar, James O Wooliscroft, (2020), Telemedicine and the Covid-19 pandemic, lessons for the future,telemedicine and eHealth,571-573.

[8] Sarah Nouri, Elaine C Khoong, Courtney R Lyles, Leah Karliner,(2020),Addressing equity in telemedicine for chronic disease management during the covid-19 pandemic,NEJM Catalyst Innovations in Care Delivery,12-15.

[9] Kimberly Lovett Rockwell, Alexis S Gilroy,(2020),Incorporating Telemedicine as part of covid-19 outbreak response systems,Am J Manag Care

[10] Jay Portnoy, Morgan Waller, Tania Elliott,(2020), Telemedicine in the era of covid19, the journal of Allergy and clinical Immunology: In Practice,1489-1491.

[11] Josep Vidal-Alaball, Ruthy Acosta-Roja,Nuria Pastor Hernandez, unai sanchez lugue,(2020), Telemedicine in the face of the covid-19 pandemic,Atencion Primaria,418-422.

[12] Zhen Hong, Nian Li, Dajang Li, Junhua Li, Bing Li, Weixi Xiong, Lu Lu, Weimin Li, Dong Zhou,(2020), Telemedicine during the covid-19 pandemic: experiences from western china, journal of medical internet research,112115. 